\title{
The Evolution of Prosthodontic Postgraduates Research in China: A Retrospective Study from A Chinese School of Stomatology
}

\author{
Wen Song*, Min Tian, Meng Meng, Lina Niu and Yumei Zhang \\ State Key Laboratory of Military Stomatology \& National Clinical Research Center for Oral Diseases \& Shaanxi Key Laboratory of Oral Diseases, Department of \\ Prosthodontics, School of Stomatology, The Fourth Military Medical University, China
}

*Corresponding author: Wen Song, State Key Laboratory of Military Stomatology \& National Clinical Research Center for Oral Diseases \& Shaanxi Key Laboratory of Oral Diseases, Department of Prosthodontics, School of Stomatology, The Fourth Military Medical University, Xi'an 710032, China.
Received Date: March 22, 2021

Published Date: April 08, 2021

\begin{abstract}
Objectives: To investigate the changes of research interests of prosthodontics. Methods: A number of 242 postgraduates' theses between 2009 and 2019 from department of prosthodontics were collected from the university library. The theses distribution by years and degree types (master/ doctor, academic/professional) were counted. The theses were categorized according to their general research areas. The top three categories were sub-divided according to their specific research contents and their distribution were determined.

Results: The academic degree percentage decreased from 2009 to 2013 and then increased, while the professional degree percentage was the opposite. The Implant category was the highest and could be sub-divided into Surface biofunctionalization (54\%), Mechanisms study (28\%) and Others. The percentage of Surface biofunctionalization was more than Mechanisms study in different degree types. However, the Mechanisms study ratio began to increase after 2014. Ceramic category was the second higher and was sub-divided into Ceramic performance (47\%), Processing technique (12\%), Metal-ceramic interaction (27\%) and Others. The Ceramic performance and Metal-ceramic interaction were the dominant in different degree types. However, after 2014, the Metal-ceramic interaction study was gradually vanished. The Adhesion was the third higher and subdivided into Mechanisms study (29\%), Application study (53\%) and Others. The Application study was more than Mechanisms study, in all different degree types. In the initial years, the Mechanisms study had not performed, and then appeared gradually.
\end{abstract}

Conclusion: The research interests of prosthodontics are closely related to the development of clinical restoration methods. The mechanisms study may grow continuously in the near future.

Keywords: Prosthodontics; Postgraduate education; Research interests; Degree thesis

\section{Introduction}

Prosthodontics is one of the traditional branches in dentistry, which pertains to the diagnosis, treatment planning, rehabilitation, and maintenance of oral function, comfort, appearance, and health of patients with clinical conditions associated with missing or deficient teeth, and/or maxillofacial tissues using biocompatible substitutes [1]. With the development of material science, new fabrication technique, and patients' requirements et al, the restoration concepts and methods may also change gradually. Unlike the undergraduate dental education that mainly focuses on the basic knowledge teaching and the most mature skills training, the postgraduate dental education focuses more on the cuttingedge frontiers and aims to cultivate investigative dentists [2]. 
Therefore, the postgraduate education of prosthodontics should also be adjusted in order to cope with the rapid progress.

On the other hand, we hypothesized that by analyzing the evolution of research directions of postgraduates' theses may help to learn the dynamic progress of prosthodontics and may also help to predict the future tendency, which is crucial for educational workers. The school of stomatology in Fourth Military Medical University (FMMU) is one of the earliest and famous dental schools in China and its prosthodontic department is the representative and predominant discipline, which enjoys its reputation both in domestic and global $[3,4]$. In the present study, we collected the postgraduate theses from our department ranging from 2009 to 2019. The theses main research interests distribution by years and degree types were analyzed, in order to find out some tendency behind.

\section{Materials and Methods}

All the postgraduate theses between 2009 and 2019 from the Department of Prosthodontics were obtained from the library of FMMU. These theses are also available from the Chinese National Knowledge Infrastructure (CNKI). In Chinese postgraduate dental education system, the master degree level is named as Master of Stomatological Medicine (SMM) while the $\mathrm{PhD}$ degree level is named as Doctor of Stomatological Medicine (SMD). Both degrees are divided into academic and professional types. Consequently, there are four different degrees, e.g., the academic SMM (SMMAcademic), professional SMM (SMM-Professional), academic
SMD (SMD-Academic) and professional SMD (SMD-Professional). The obtained theses were sorted by date and then sub-divided by different degrees. In the next, the theses were categorized by different research interests (first level categorization), based on the main contents that estimated from the title, key words and abstract.

From the first level categorization, we obtained three main directions, e.g., the Implant, Ceramics and Adhesion, which were analyzed in detail. The Implant category was sub-divided into Surface biofunctionalization, Mechanisms study and Others. The Ceramic category was sub-divided into Ceramic performance, Processing technique, Metal-ceramic interaction and Others. The Adhesion category was sub-divided into Mechanisms study, Application study and Others. The three main categories and their sub-categories distribution by year and degree types were calculated.

\section{Results}

\section{Whole theses list analysis}

A total number of 242 theses were collected, in which contained 63 SMD-Academic, 25 SMD-Professional, 83 SMM-Academic and 71 SMM-Professional. From 2009 to 2019, the number of theses was gradually increased above 20 and in 2013 reached the peak of 36. Then the number decreased gradually below 20. From 2009 to 2011, the ratio of professional degree was very few, both in SMD and SMM. Then the ratio increased rapidly and reached the equivalent level to academic degree (Figure 1A).
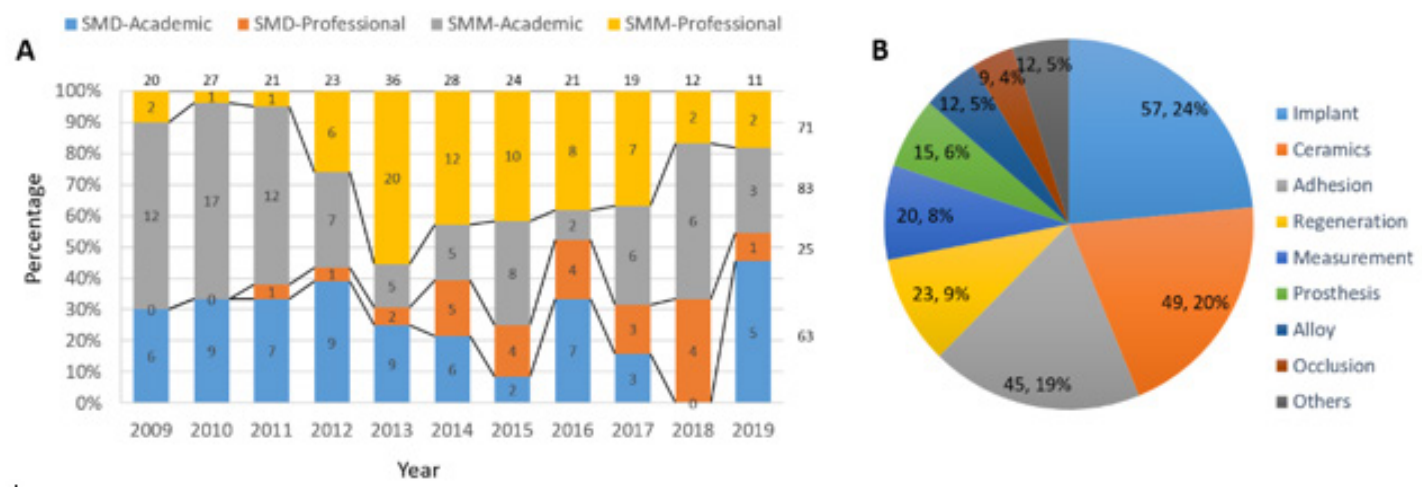

Figure 1: General information of the whole theses list.

Figure 1A: The theses percentage of different degree types in each year. The numbers in the bar show exact thesis number. The numbers above the bar show the sum of each year while the numbers at right show the sum of each degree type.

Figure 1B: The percentage of different research interests. The labels are the exact theses number and relative percentage.

The whole theses list was categorized by different research interests (Figure 1B). The mostly studied projects were Implant (24\%), Ceramics (20\%) and Adhesion (19\%). These would be analyzed in detail afterwards. The Regeneration direction occupied 9\%, which mainly contained the mesenchymal stem cells biology, functional scaffold materials, bone regeneration and vascularization. The Measurement study was $8 \%$, which roughly indicated the study of tooth morphology measurement, prostheses bio-mechanical measurement and some measurement methods. The Prostheses indicated the study of maxillofacial defects restoration that mainly contained cleft palate therapy, silicone rubber and maxillofacial organs 3D database collection. The Alloy category focused on titanium-based metal processing, 3D printing and antibacterial metals. The Occlusion study mainly 
contained investigation of occlusal attrition, occlusal interference and periodontal tissues health.

\section{Implant category analysis}

In the Implant category, the percentages of SMD-Academic, SMD-Professional, SMM-Academic and SMM-Professional were $30 \%, 14 \%, 26 \%$ and 30\% respectively (Figure 2A). The category was further divided into Surface biofunctionalization, Mechanisms study and Others, which occupied $54 \%, 26 \%$ and $18 \%$ respectively (Figure 2B). The Surface biofunctionalization mainly focused on titanium implant surface modification such as micro arc oxidation, anodization, biomolecules immobilization, and antibiotics coating, in order to obtain enhanced osteogenesis and/or anti-bacteria properties.
A
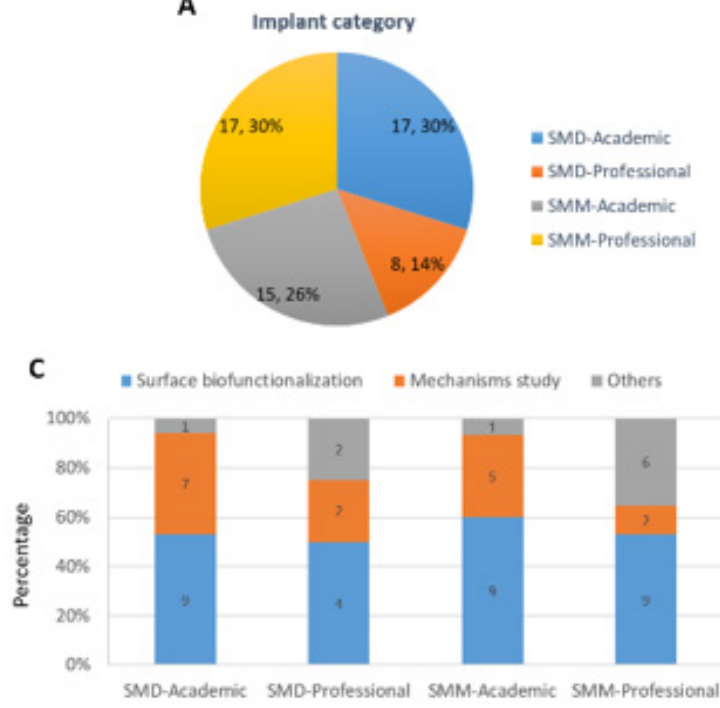

B

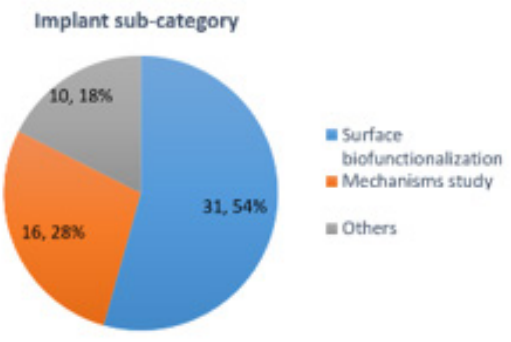

D

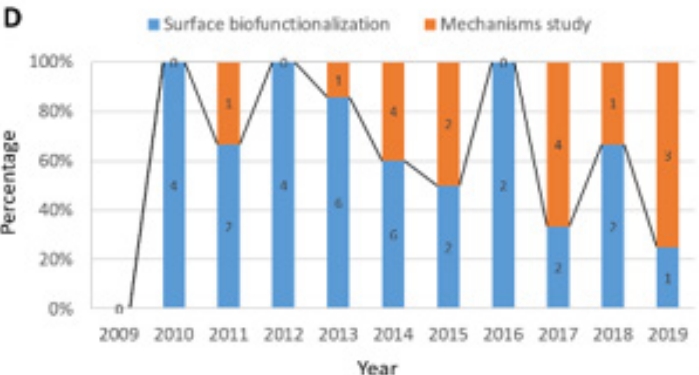

Figure 2: The Implant category analysis.

Figure 2A: The Implant category theses distribution by different degree types. The labels are the exact theses number and relative percentage.

Figure 2B: The sub-category research directions distribution. The labels are the exact theses number and relative percentage.

Figure 2C: The sub-category theses percentage in each degree type. The numbers in the bar show exact thesis number.

Figure 2D: The sub-category theses percentage in each year. The numbers in the bar show exact thesis number.

The Mechanisms study mainly focused on how the implant surface properties can influence the biological functions. Some porous titanium implant research and clinical relevant studies were left to the Others sub-category. In SMD-Academic, the Surface biofunctionalization and Mechanisms study were nearly the same, and only 1 theses was the Others sub-category. Similar ratio was obtained in SMM-Academic. In SMD-Professional and SMMProfessional, the Surface biofunctionalization sub-category was $\sim 50 \%$, while the Mechanisms study ratio was decreased (Figure 2C). The Implant research began from 2010, when there was no Mechanism study. Then the Mechanisms study ratio increased gradually and became dominant in recent years (Figure 2D).

\section{Ceramic category analysis}

In the Ceramic category, the percentages of SMD-Academic, SMD-Professional, SMM-Academic and SMM-Professional were $18 \%, 6 \%, 47 \%$ and $29 \%$ respectively (Figure $3 A$ ). The category was further divided into Ceramic performance, Processing technique, Metal-ceramic interaction and Others, which occupied 47\%,12\%, $27 \%$ and $14 \%$ respectively (Figure 3B). The Ceramic performance was mainly about the wear resistance, adhesive property and aesthetic property. The Processing technique mainly focused on porcelain machining such as laser rapid fabrication and dental hear-pressing, in order to improve clinical behaviors.

The Metal-ceramic interaction study was trying to understand the mechanisms of interaction between porcelain and pure titanium or Ni-Cr alloy, and how to improve the affinity. In SMD-Academic, the Ceramic performance and Processing technique were roughly similar, while the Metal-ceramic interaction accounted for more than $40 \%$. In SMD-Professional, there were 2 theses on Ceramic performance and 1 theses on Metal-ceramic interaction. In SMMAcademic, nearly half was about Ceramic performance. The Processing technique and Metal-ceramic interaction accounted for $\sim 10 \%$ and $\sim 20 \%$ respectively. In SMM-Professional, nearly $60 \%$ was about Ceramic performance and $\sim 20 \%$ was about Metalceramic interaction (Figure 3C). Generally, the Ceramic performance study and the Metal-ceramic interaction study have similar theses number each year from 2009 to 2014, except in 2011 that the 2 theses were all about Metal-ceramic interaction. Afterwards, except in 2016, all the theses were about Ceramic performance. 
A
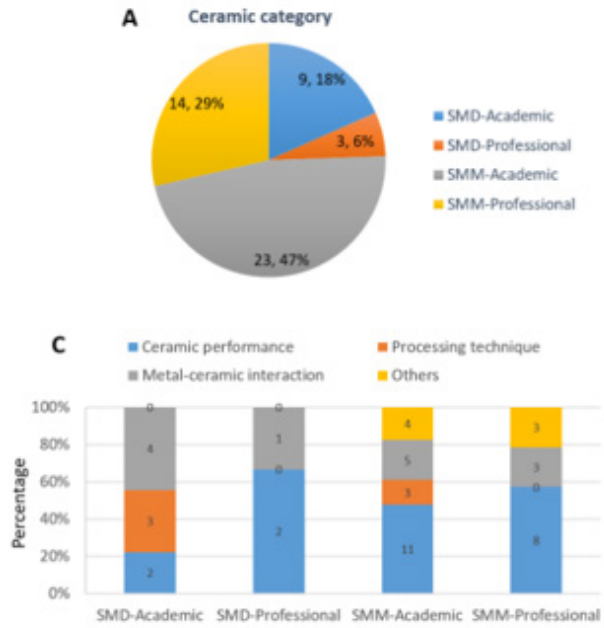

B
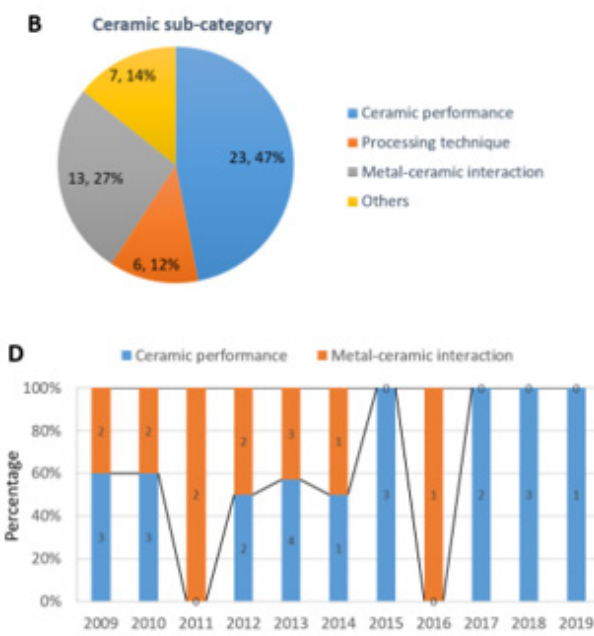

Year

Figure 3: The Ceramic category analysis.

Figure 3A: The Ceramic category theses distribution by different degree types. The labels are the exact theses number and relative percentage. Figure 3B: The sub-category research directions distribution. The labels are the exact theses number and relative percentage.

Figure 3C: The sub-category theses percentage in each degree type. The numbers in the bar show exact thesis number.

Figure 3D: The sub-category theses percentage in each year. The numbers in the bar show exact thesis number.

\section{Adhesion category analysis}

In the adhesion category, the degree types distribution was 40\%, 13\%, 29\% and 18\% respectively for SMD-Academic, SMDProfessional, SMM-Academic and SMM-Professional (Figure $4 \mathrm{~A})$. The category was further divided into Mechanisms study,
Application study and Others, which occupied 29\%, 53\% and $18 \%$ respectively (Figure 4B). The Mechanism study was mainly about the influence of dentin biology on adhesion bonding. The Application study mainly focused on developing multifunctional and strengthened bonding agent. There were also some resin optimization theses, which were left to the Others sub-category.
A

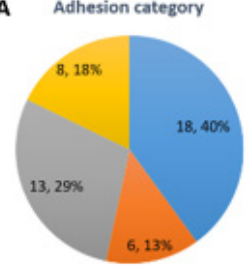

asMD-Professional - SMM-Academic =SMM-Professional
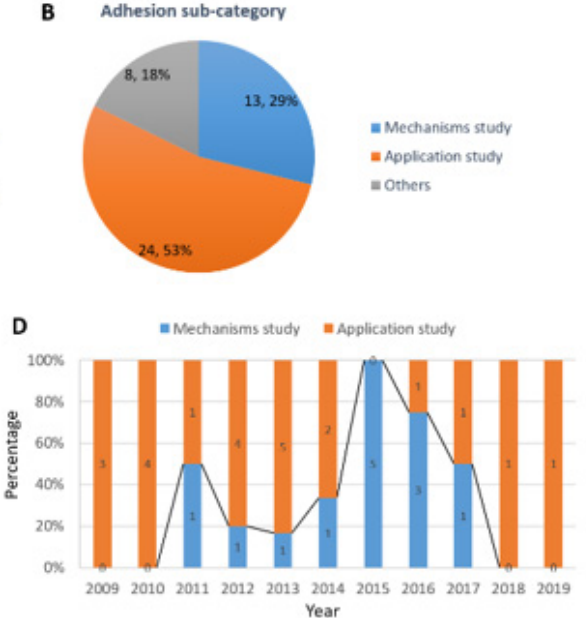

Figure 4: The Adhesion category analysis.

Figure 4A: The Adhesion category theses distribution by different degree types. The labels are the exact theses number and relative percentage.

Figure 4B: The sub-category research directions distribution. The labels are the exact theses number and relative percentage.

Figure 4C: The sub-category theses percentage in each degree type. The numbers in the bar show exact thesis number.

Figure 4D: The sub-category theses percentage in each year. The numbers in the bar show exact thesis number.

In SMD-Academic, the Mechanisms study was $~ 30 \%$ while the Application study was $\sim 40 \%$. In SMD-Professional, the Mechanisms study was $\sim 50 \%$ while the Application study was $\sim 30 \%$. In SMM-Academic and SMM-Professional, the Application study was dominant, which was $\sim 3$ folds of the Mechanisms study (Figure 4C). Generally, the Application study was the main direction 
throughout the decade. Specifically, the Mechanisms study began to emerge in 2011 and remained at a low ratio. From 2015 to 2017, the Mechanisms study ratio was no less than the Application study. Afterwards, there were only Application studies again (Figure 4D).

\section{Discussion}

The postgraduate dental degrees are various around the world. There are a number of postgraduate dental degrees such as Master of Dental Surgery (MDS), Master of Stomatology (MS), Doctor of Philosophy in Dentistry (PhD), Doctor of Dental Surgery (DDS) and Doctor of Dental Medicine (DMD). From 1999 in China, the postgraduate dental degree names have been standardized as Master of Stomatological Medicine (SMM) and Doctor of Stomatological Medicine (SMD), and both have two types of academic and professional [5]. The academic postgraduate education put more emphasis on fundamental research, aims to cultivate scientific research workers. The professional postgraduate education emphasizes more on clinical research, aims to cultivate specific technicians [2,6]. It is clear that from 2009 on, the professional degrees ratio grows rapidly both in SMD and SMM, which may be due to policy encouragement on professional education and the clinical employment requirements [7]. Another trend is that the total SMD ratio gradually increase from $30 \%$ to $50 \%$, suggesting that a more higher education is in demand.

The research directions of prosthodontics are closely related to the development of material science and new basic mechanisms revealed. We have roughly classified the theses into different research directions, according to their main contents. After statistics, the Implant, Ceramics and Adhesion are the top three categories, which exactly fit the characteristics of fixed prosthodontics that the crowns/bridges are cemented onto teeth or implants [8]. The dental implant grows rapidly recent years due to its various advantages such as adjacent teeth preservation, high masticatory efficiency and comfortable to use [9]. Therefore, the implant studies become more and more popular in postgraduates' theses.

We may infer that this tendency will still last for several years since the dental implant restoration has not been fully popularized yet in China. The primary goal of dental implant is the rapid osseointegration establishment, and therefore the Surface biofunctionalization occupied more than $50 \%$, no matter in which kind of degree types. From the annual theses data, in the early years, there are few studies about Mechanisms study, which however, becomes dominant after 2017. This may imply that people are aware of the importance of understanding the mechanisms behind, after numerous surface decoration studies. In addition, with time passing by, the peri-implant infection is more vulnerable and we believe that the anti-bacterial strategy may obtain more and more concerns.

The introduction of zirconium dioxide and aluminium oxide based ceramic materials into prosthodontics is a great success for restorations fabrication such as inlay, onlay, veneer, full crown, and fixed bridges. In Ceramic category, the SMM postgraduates are much more than SMD and nearly half of the Ceramic studies are Ceramic performance observation. This suggests that the observational and descriptive studies are more suitable for master degree candidates. Once upon a time, the porcelain-fused-to-metal (PFM) is considered as an ideal material for crown fabrication, which possesses both the strength of metal and the aesthesis of porcelain [10]. As a result, there are a number of studies about Metal-ceramic interaction, particularly before 2017. However, with the improvement of allceramic materials, the PFM is replaced gradually and the Metalceramic interaction studies are no longer meaningful anymore. We infer that the strength, aesthesis and bonding properties of allceramic materials may still be the important research area in the near future. However, some new sub-categories such as the gingival fibroblasts compatible surface may draw more attentions, for the reason that there are already several reports about fibroblasts culture on ceramic surface $[11,12]$, but no thesis found here in our department.

The adhesives are extensively used in clinic and nearly all the fixed restorations such as inlay, only, veneer and full crowns require rigid adhesion. It is an old and yet new story in prosthodontics. With the emerging of dental resin for caries filling, the development of resin-based adhesives is initiated and new generation appears continuously [13]. In the Adhesion category, the Application study takes 53\% percent, which is nearly twice over Mechanisms study. More specifically, in SMD degrees, the Application study vs Mechanisms study ratio is not significantly different, whereas in the SMM degrees the Application study ratio is remarkably increased. This may indicate that the master degree candidates are more opt for material screening and optimization, whereas the doctoral candidates are trying to figure out the mechanisms behind. In most years, the theses of Application study are superior, aiming to find out more powerful and multifunctional adhesives. However, the new bonding theory should not be neglected because new theory often brings revolutionary changes. For example, Sui Mai, et al. [14] has reported a new concept of chelate-and-rinse dentin bonding theory, which may bring the gap between wet and dry dentin bonding [14]. Therefore, the Mechanism study may attract more and more attention in the following stage.

\section{Conclusion}

By analyzing the research alterations of postgraduates' theses in the last decade from our department, we have found that the dental implant, ceramic materials and dentin bonding are the top fields studied. Specifically, the implant surface biofunctionalization, ceramic physical performances and multifunctional adhesives are the main subjects. However, in the near future, the cell response mechanisms to implant surface, ceramic biological properties and dentin bonding mechanisms may draw more and more attention. 


\section{Discloure}

No potential competing interests disclosed.

\section{Acknowledgement}

None.

\section{References}

1. Zhao J, Wang X (2014) Chapter 3-Dental Prostheses. In: Shen JZ, Kosmač T (Eds.) Advanced Ceramics for Dentistry. Oxford: ButterworthHeinemann, pp: 23-49.

2. Zheng JW, Zhang SY, Yang C (2013) Current Undergraduate and Postgraduate Dental Education in China. J Dent Educ 77(1): 72-78.

3. Yang J, Zhang Y, Ye X (2014) Dental education evaluation in China: a systematic review. BMC Med Educ 14: 178.

4. Jia TANG TS (2015) A brief introducation of dental education in mainland China. The Dental Journal of Health Sciences University of Hokkaido 34(1): 1-12.

5. Zhonghua Kou, Qiang Yi Xue, Za Zhi (2013) National Symposium on Postgraduate Education in Stomatology 48(08): 510-511.

6. Wang YH, Zhao Q, Tan Z (2017) Current differences in dental education between Chinese and Western models. Eur J Dent Educ 21(4): e43-e49.

7. Wu ZY, Zhang ZY, Jiang XQ, Guo L (2010) Comparison of dental education and professional development between mainland China and North America. Eur J Dent Educ 14(2): 106-112.
8. Fujimoto J (2006) Contemporary Fixed Prosthodontics. In: Rosenstiel S, Land M (Eds.), (4 ${ }^{\text {th }}$ Edn.).

9. Hebel K, Gajjar R, Hofstede T (2000) Single-tooth replacement: bridge vs. implant-supported restoration. J Can Dent Assoc 66(8):435-438.

10. Gallucci GO, Grutter L, Nedir R, Bischof M, Belser UC (2011) Esthetic outcomes with porcelain-fused-to-ceramic and all-ceramic singleimplant crowns: a randomized clinical trial. Clin Oral Implants Res 22(1): 62-69.

11. Rizo Gorrita M, Herráez Galindo C, Torres Lagares D, Serrera Figallo MÁ, Gutiérre Pérez JL (2019) Biocompatibility of Polymer and Ceramic CAD/CAM Materials with Human Gingival Fibroblasts (HGFs). Polymers 11(9): 1446.

12. Pae A, Lee H, Kim HS, Kwon YD, Woo YH (2009) Attachment and growth behaviour of human gingival fibroblasts on titanium and zirconia ceramic surfaces. Biomed Mater 4(2): 025005.

13. Sofan E, Sofan A, Palaia G, Tenore G, Romeo U, et al. (2017) Classification review of dental adhesive systems: from the IV generation to the universal type. Ann Stomatol (Roma) 8(1): 1-17.

14. Mai S, Wei CC, Gu LS (2017) Extrafibrillar collagen demineralizationbased chelate-and-rinse technique bridges the gap between wet and dry dentin bonding. Acta Biomater 57: 435-448. 